\title{
Covert attention in touch: Behavioral and ERP evidence for costs and benefits
}

\author{
BETTINA FORSTER AND MARTIN EIMER \\ Birkbeck College, University of London, Malet Street, London, United Kingdom
}

\begin{abstract}
To investigate the mechanism underlying tactile spatial attention, reaction times (RTs) and event-related potentials (ERPs) were recorded in response to mechanical stimuli delivered to the hands. At the start of each trial cues indicated either the correct (valid) or incorrect (invalid) tactile stimulus location or were uninformative (neutral). RT costs (suppression of invalid compared to neutral trials) were found to be larger than benefits (enhancement of valid compared to neutral trials). ERPs showed that costs and benefits contribute equally to attentional modulations of the somatosensory N140 component, whereas these were largely due to costs at longer latencies. These results differ from the pattern of attentional ERP modulations previously found for vision and audition, where costs precede benefits, and therefore suggest that the mechanisms of attentional selectivity in touch might be different from attentional processes in other modalities.
\end{abstract}

Descriptors: Tactile spatial attention, Costs, Benefits, Reaction times (RTs), Event-related potentials (ERPs)

The capacity to shift the focus of attention to different locations in the absence of overt eye or head movements (covert spatial attention) is central to our ability to select relevant objects and events. A popular behavioral task to assess the effects of such covert shifts of spatial attention was introduced by Posner (1978). In this task, participants are required to detect targets presented to the left or right of fixation. Prior to each target, a centrally located cue is presented that provides information about the likely location of an upcoming target. In the majority of trials, the cue is valid as it indicates the correct target location. However, on some trials the cue is invalid as it indicates the incorrect target location. Comparing reaction times (RTs) and accuracy measures on valid and invalid trials has revealed superior behavioral performance in response to targets at validly cued locations. This effect of prior knowledge of likely target positions is seen as a result of covert shifts of attention to expected target locations. To further assess whether this attention directing effect is due to enhanced target processing at cued locations ("benefits") or suppression of target processing at uncued locations ("costs"), performance on valid and invalid trials can be compared to performance in neutral trials, where cues provide no information about the subsequent target location. On neutral trials, spatial attention is assumed to be unfocused or divided between possible target locations. Benefits of directing spatial attention are measured by comparing performance on valid trials to neutral trials, whereas costs are revealed by comparing per-

This research was supported by a grant from the Biotechnology and Biological Research Council (BBSRC).

Address reprint requests to: Bettina Forster, Department of Psychology, City University, Northampton Square, London EC1V0HB, U.K. E-mail: b.forster@city.ac.uk. formance on invalid trials to neutral trials. Thus, it can be assessed whether the overall attention directing effect is due to costs, benefits, or a combination of both.

Behavioral effects of covert spatial attention effects have been well documented for vision and audition. These effects are seen in speeded RTs and improved discrimination on valid trials in response to targets at correctly cued locations as compared to targets presented after an invalid cue. In addition, several studies have also evaluated the contribution of costs and benefits to these behavioral effects of covert spatial attention in vision (e.g., Luck et al., 1994; Posner, 1978) and audition (e.g., Schröger \& Eimer, 1997; Spence \& Driver, 1997) by comparing behavioral performance on valid and invalid trials to performance on neutral trials. RTs were found to be fastest when visual or auditory stimuli were presented at cued locations (valid trials), intermediate to stimuli after neutral cues, and slowest to stimuli at uncued locations (invalid trials). These findings demonstrate that in vision and in audition, shifts of covert spatial attention result in an enhancement of information processing at attended locations (reflected in performance benefits on valid trials) as well as in a suppression of processing at unattended locations (resulting in performance costs on invalid trials).

In contrast to visual and auditory attention, the effects of covert shifts of spatial attention on somatosensory processing are less well documented. Although some studies have shown that tactile-spatial attention can facilitate accuracy (Sathian \& Burton, 1991) and discrimination speed (Spence, Pavani, \& Driver, 2000) in touch, the question of whether these effects reflect costs, benefits, or a combination of both has not yet been systematically investigated. The only evidence so far comes from an experiment reported by Posner (1978), which investigated effects of spatial attention in a task where participants had to discriminate low 
intensity targets and higher intensity nontargets delivered to their right and left index fingers. A neutral cue was shown on half of all trials, whereas on the other trials informative cues were presented that indicated the location of an upcoming tactile event correctly on $80 \%$ of these trials (valid trials) and incorrectly on $20 \%$ (invalid trials). RTs were found to be fastest on valid trials, slowest on invalid trials, and intermediate on neutral trials. However, no formal statistical analyses of these differences were reported. This pattern of results suggests that, analogous to vision and audition, shifts or tactile attention also result in behavioral benefits as well as costs. In contrast, Posner (1978) found that tactile attention had no effects on performance when the tactile task did not involve stimulus discrimination, but just simple detection of a tactile event (but see Spence et al., 2000, for a critical evaluation of this null effect).

Event-related brain potentials (ERPs) have proved to be extremely useful to investigate the relative contributions of the costs and benefits of spatial attention on the processing of sensory stimuli. In vision, Luck et al. (1994) found a reduction of P1 amplitudes in response to visual stimuli on invalid trials (i.e., stimuli presented at uncued locations) relative to visual stimuli on neutral trials. This effect was followed by an enhancement of the N1 component on valid trials (i.e., in response to visual stimuli at correctly cued locations) relative to neutral trials. Importantly, no P1 enhancement was found for valid trials, and no N1 suppression was present on invalid relative to neutral trials. This pattern of results suggests that processing costs (resulting in a suppression of the P1 component) and benefits (reflected by an enhancement of the $\mathrm{N} 1$ component) affect separate stages of visual processing and that costs appear earlier than benefits. A similar time course of costs and benefits of spatial attention was observed in the auditory modality. Golob, Pratt, and Starr (2002) and Schröger and Eimer (1997) reported ERP differences for invalid compared to neutral trials (costs) for the N100/P200, followed by enhanced negativities for valid compared to neutral trials at longer latencies (benefits). These studies suggest that in both vision and audition, costs precede benefits. Shifts of spatial attention initially lead to a suppression of sensory processing at unattended locations, and only later result in enhancement of processing at attended locations.

A number of ERP studies have also investigated effects of spatial attention on somatosensory processing by comparing ERPs triggered by tactile stimuli at attended versus unattended locations (e.g., Desmedt \& Robertson, 1977; Eimer \& Forster, 2003; Forster \& Eimer, 2004; García-Larrea, Lukaszewicz, \& Mauguière, 1995; Michie, Bearpark, Crawford, \& Glue, 1987). In most of these studies, attention was found to result in enhancements of the somatosensory N140 component followed by a sustained negativity at longer latencies, although modulatory effects on earlier components (N80, P100) have also been observed (Eimer \& Forster, 2003; Josiassen, Shagrass, Roemer, Ercegovac, \& Straumanis, 1982; Michie, 1984). Surprisingly, not a single ERP study to date has attempted to dissociate the costs and benefits of spatial attention on somatosensory processing. The aim of the present experiment was to fill this gap in our knowledge by studying for the first time whether the effects of tactile-spatial attention on behavioral performance and on somatosensory ERP components reflect distinct and separable contributions of both processing costs and benefits.

In our experiment, single vibratory tactile stimuli were delivered to the left or right hand. Participants had to respond vocally whenever a target stimulus (a slightly weaker vibration) was detected and to refrain from responding on trials where a nontarget (a slightly stronger vibration) was presented. Symbolic visual precues either signaled the side of an upcoming target stimulus with $80 \%$ validity, or gave no information with respect to target stimulus side. Task-irrelevant nontargets were presented with equal probability on the cued and uncued sides.

RTs were measured on trials where task-relevant tactile target stimuli were presented. Costs and benefits of tactile-spatial attention were evaluated by comparing RTs on neutral trials to RTs on invalid and valid trials, respectively. On the basis of Posner's (1978) informal observations, we expected to find behavioral costs as well as benefits. We also assessed whether there are any differences in the relative contributions of costs and benefits to the overall behavioral effects of tactile-spatial attention. ERPs were computed for nontarget trials only, to avoid any contamination with artifacts related to vocal responding. Overall effects of spatial attention were evaluated by comparing ERPs triggered on valid and invalid trials. In line with earlier observations (Desmedt \& Robertson, 1977; Eimer \& Forster, 2003; Forster \& Eimer 2004; García-Larrea et al., 1995; Michie et al., 1987), we expected to find attentional modulations of the N140 component followed by a subsequent sustained attentional negativity. Most importantly, we compared somatosensory ERPs elicited on valid and invalid trials to ERPs recorded on neutral trials in order to assess the presence and the time course of costs and benefits of tactile-spatial attention. If the pattern of ERP effects previously observed in vision and audition also applied to touch, processing costs should appear earlier than benefits. In other words, ERP evidence indicative of the suppression of tactile processing at unattended locations (obtained by comparing ERPs recorded on invalid and neutral trials) should be present before any differences in ERP waveforms between valid and neutral trials (reflecting enhanced processing at attended locations) are triggered.

\section{Methods}

\section{Participants}

Sixteen paid volunteers participated in this experiment. Three participants were excluded due to poor eye fixation control (see below) and 1 participant due to an excessive amount of eye blinks, so that 12 participants (6 women, 6 men, aged 23-34 years, mean age 29 years) remained in the sample. All participants were right-handed and had normal or corrected-to-normal vision by self-report.

\section{Stimuli and Apparatus}

Participants sat in a dimly lit experimental chamber, wearing a head-mounted microphone. Cues consisted of symmetrical stimulus arrays made up of two adjacent triangles, which were presented centrally on a computer screen at a viewing distance of 55 $\mathrm{cm}$ (total visual angle covered: $3.5^{\circ} \times 2.5^{\circ}$; visual angle of each triangle: $1.2^{\circ} \times 2.5^{\circ}$ ). The two triangles were both red (luminance: $14 \mathrm{~cd} / \mathrm{m}^{2}$ ), or both blue (luminance: $19 \mathrm{~cd} / \mathrm{m}^{2}$ ), or one triangle was red and the other triangle was blue. They always pointed in opposite directions ( $\backslash$ or $\backslash$ ). A fixation cross, located between both triangles, was continuously present.

Tactile stimuli were presented using two 5-V solenoids, driving a metal rod with a blunt conical tip to the radial side of the middle phalanx of the left and right index fingers, making contact with a finger whenever a current was passed through the solenoid. The 
solenoids were attached to the fingers with medical tape. White noise (65 dB SPL, measured from the position of the participants' head) was continuously present to mask any sounds made by the tactile stimulators. Tactile stimuli were vibrations, and these were generated by presenting a rapid sequence of 20 brief pulses. The stimulus onset asynchrony between successive pulses was $17 \mathrm{~ms}$, corresponding to a rectangular stimulation frequency of $58.8 \mathrm{~Hz}$. Tactile vibrations differed with respect to their intensity. To present "weak" vibrations (which served as target stimuli), the contact time between rod and skin was set to $2 \mathrm{~ms}$, followed by a 15 -ms interpulse interval. To present "strong" vibrations (which were nontarget stimuli), contact time was set to 3 $\mathrm{ms}$, followed by a 14-ms interpulse interval. Subjectively, these manipulations resulted in perceived vibrations with identical frequencies, but different intensities. The duration of each vibratory stimulus (the interval between the onset of the first pulse and the offset of the last pulse) was $325 \mathrm{~ms}$ (for weak vibrations) or 326 ms (for strong vibrations).

\section{Procedure}

The experiment consisted of 12 blocks, with 78 trials per block. Each trial started with a $100-\mathrm{ms}$ presentation of a visual cue. At $600 \mathrm{~ms}$ after cue offset, a tactile stimulus was presented unilaterally to the left or right hand. The intertrial interval was 1000 ms. Fifty-two trials per block started with a directional cue, which indicated the likely side where a target stimulus would occur. Directional cues were composed of one red triangle and one blue triangle, and the likely target side was signaled by the direction of one of these triangles. For 6 participants, blue triangles were relevant (i.e., cues containing a left-pointing blue triangle indicated that tactile targets would be more likely on the left than on the right side, whereas cues containing a right-pointing blue triangle indicated the right side as likely target location). Red triangles were relevant for the other 6 participants. Relevant left-pointing or right-pointing triangles were presented with equal probability to the left or right of fixation. In the remaining 26 neutral trials per block, nondirectional cues were presented, which consisted of two triangles of the same (relevant) color. Because the two triangles always pointed in opposite directions, no advance information about the likely target location was provided by these nondirectional cues.

Tactile targets (which required a vocal response) were delivered in 30 trials per block. Tactile targets were preceded by directional cues on 20 of these trials. On 16 valid target trials, a tactile target appeared at the location indicated by the cue (eight left targets and eight right targets), and only on four invalid target trials was a tactile target presented on the uncued side. Thus, the validity of directional cues was $80 \%$ for target trials. Tactile targets were preceded by neutral cues on 10 trials per block (five left targets and five right targets). Tactile nontarget stimuli were presented in 48 trials per block. They were preceded by directional cues on 32 of these trials (eight trials for each of the four combinations of cued location and stimulus location). Thus, while directional cues were informative with respect to target side, they provided no information with respect to the side of task-irrelevant nontargets. In 16 trials per block, tactile nontargets were preceded by nondirectional cues (eight left nontargets and eight right nontargets).

Participants were instructed to place their hands on a table 26 $\mathrm{cm}$ apart, to keep their gaze focused on the central fixation cross, to respond vocally ("yes") whenever a target stimulus (a weak vibration) was detected, and to ignore all tactile nontarget stimuli (strong vibrations). Vocal response latencies were measured with a voice key. Participants were informed that informative cues would indicate the likely location of target stimuli and were encouraged to use this advance information in order to improve their performance. To eliminate any visual information about hand and arm position, participants' hands and forearms were placed under a second table top, and upper arms were covered by a cloth attached to the second table top (see also Eimer, Van Velzen, Forster, \& Driver 2003). Participants were monitored continuously with a video camera.

\section{Recording and Data Analysis}

EEG was recorded with $\mathrm{Ag}-\mathrm{AgCl}$ electrodes and linked-earlobe reference from FPz, F7, F3, Fz, F4, F8, FC5, FC6, T7, C3, Cz, C4, T8, CP5, CP6, P7, P3, Pz, P4, P8, and Oz (according to the 10-20 system), and from OL and OR (located halfway between $\mathrm{O} 1$ and $\mathrm{P} 7$, and $\mathrm{O} 2$ and $\mathrm{P} 8$, respectively). Horizontal EOG was recorded bipolarly from the outer canthi of both eyes. Electrode impedance was kept below $5 \mathrm{k} \Omega$, and the impedances of the earlobe electrodes were kept as equal as possible. Amplifier bandpass was $0.1-40 \mathrm{~Hz}$, and digitization rate was $200 \mathrm{~Hz}$.

EEG and EOG were epoched off-line into 1200 -ms periods, starting $100 \mathrm{~ms}$ prior to cue onset and ending $400 \mathrm{~ms}$ after the onset of a tactile stimulus. Trials where horizontal eye movements (HEOG exceeding $\pm 30 \mu \mathrm{V}$ relative to baseline) were detected throughout this period (usually indicating an eye movement toward the cued side) were excluded from analysis. Likewise, trials with eye blinks (FPz exceeding $\pm 60 \mu \mathrm{V}$ relative to baseline) or other artifacts (a voltage exceeding $\pm 60 \mu \mathrm{V}$ at any electrode location relative to baseline) obtained in the $400-\mathrm{ms}$ interval following tactile stimulus onset were excluded from analysis. Averaged HEOG waveforms obtained in the interval between cue onset and $400 \mathrm{~ms}$ after the onset of the peripheral tactile stimulus were scored for systematic deviations of eye position, indicating a tendency to move the eyes toward the cued side. Two participants were disqualified due to residual HEOG deflections exceeding $\pm 3 \mu \mathrm{V}$ in the cue-target interval, and 1 participant was excluded because of HEOG deflections exceeding this criterion after tactile stimulus onset.

Analyses of EEG data were only conducted for ERPs obtained in response to tactile nontarget stimuli (strong vibrations). EEG was averaged relative to a 100 -ms prestimulus baseline for all combinations of trial type (valid vs. invalid vs. neutral) and stimulated hand (left vs. right). ERP mean amplitudes were computed within successive measurement windows centred on the latencies (in milliseconds poststimulus) of somatosensory P100 (80-120 ms) and N140 (120-165 ms) components. To investigate longer latency effects of attention, mean amplitudes were also computed between $175 \mathrm{~ms}$ and $285 \mathrm{~ms}$ poststimulus. Statistical analyses of ERP mean amplitudes were conducted for lateral recording sites where the amplitudes of early somatosensory components are maximal (FC5/6, C3/4, CP5/6, and P3/4), as well as for midline electrodes $\mathrm{Fz}, \mathrm{Cz}$, and $\mathrm{Pz}$. Mean amplitude values were analyzed with repeated-measures ANOVA, separately for midline electrodes and for electrodes contralateral and ipsilateral to the anatomical side of the stimulated hand.

Three sets of analyses were conducted to investigate (a) overall effects of tactile-spatial attention (by contrasting ERPs on valid and invalid trials), (b) any benefits of tactile attention (by contrasting ERPs on valid and neutral trials), and (c) any costs of tactile attention (by contrasting ERPs on neutral and invalid trials). These analyses included the factors trial type (valid vs. 
invalid, valid vs. neutral, neutral vs. invalid), stimulated hand (left vs. right), and electrode site (FC5/6 vs. C3/4 vs. CP5/6 vs. P3/4 for contralateral and ipsilateral electrodes; Fz vs. Cz vs. Pz, for midline sites). When appropriate, Greenhouse-Geisser adjustments to the degrees of freedom were applied, and the corrected $p$ values are reported. Additional cost-benefit analyses were conducted to assess the relative contributions of costs and benefits to the attentional modulations of somatosensory ERPs. In these analyses, benefits and costs were calculated by subtracting mean ERP amplitudes on valid trials from ERPs on neutral trials and ERPs on neutral trials from ERPs on invalid trials, respectively.

For vocal response times, repeated-measures ANOVAs were performed for the factors trial type (valid vs. invalid vs. neutral) and stimulated hand (left vs. right). To assess behavioral costs and benefits of tactile-spatial attention, paired $t$ tests were used to compare RTs on valid and neutral trials and neutral and invalid trials, respectively. Finally, the size of RT benefits (obtained by subtracting RTs on neutral trials from RTs on valid trials) and costs (obtained by subtracting invalid trial RTs from neutral trial RTs) was directly compared via paired $t$ tests.

\section{Results}

\section{Behavioral Performance}

Vocal responses to tactile target stimuli differed as a function of trial type (valid vs. invalid vs. neutral), $F(2,22)=62.4, p<.01$, $\varepsilon=.60$, with fastest RTs to targets on valid trials, slowest RTs on invalid trials, and intermediate RTs on neutral trials (mean RTs of $487 \mathrm{~ms}, 631 \mathrm{~ms}$, and $527 \mathrm{~ms}$, respectively, corresponding to benefits of $40 \mathrm{~ms}$ and costs of $104 \mathrm{~ms}$; see Figure 1). Follow-up analysis revealed that both benefits and costs were statistically significant, both $t(11)>4.6$, both $p<.01$. An additional analysis, which contrasted the size of RT benefits (obtained by subtracting $\mathrm{RTs}$ on neutral trials from RTs on valid trials) and RT costs (obtained by subtracting RTs on invalid trials from RTs on neutral trials) revealed that costs were significantly larger than benefits, $t(11)=6.0, p<.001$.

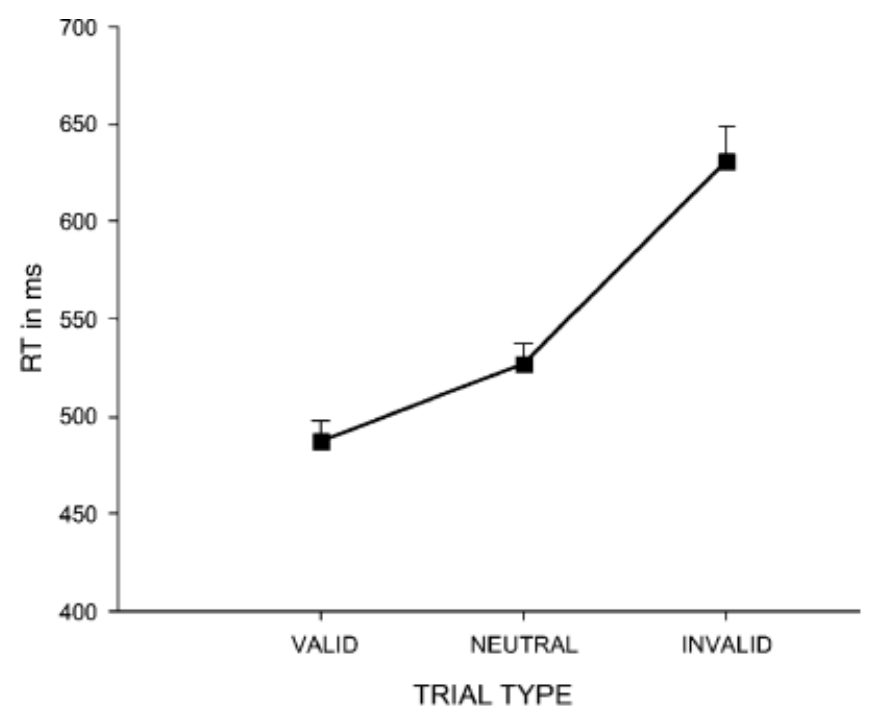

Participants missed $1.6 \%$ of all tactile targets. The percentage of missed targets on valid, invalid, and neutral trials $(1.0 \%$, $1.7 \%$, and $3.6 \%$, respectively) did not differ significantly. False alarms to tactile nontargets occurred on less than $1 \%$ of those trials.

\section{Somatosensory Evoked Potentials}

Overall effects of tactile-spatial attention: Valid and invalid trials. Figure 2 shows somatosensory evoked potentials (SEPs) in response to tactile nontarget stimuli observed at midline electrodes and at electrodes ipsilateral and contralateral to the stimulated hand on valid trials (solid lines) and on invalid trials (dotted lines). Because there were no significant main effects or interaction involving the factor stimulated hand, SEPs are collapsed across left-hand and right-hand stimulation in this as well as in subsequent figures. Effects of tactile-spatial attention are apparent as an enhanced negativity for tactile stimuli on valid trials. This attentional modulation started at the peak of the P100 component, resulted in enhanced N140 amplitudes, and was still present at longer latencies. In the P100 latency window (80-120 ms after stimulus onset), a main effect of trial type (valid vs. invalid) was present at midline electrodes, $F(1,11)=13.2, p<.01$, and at ipsilateral electrodes, $F(1,11)=6.0, p<.04$, reflecting an enhanced negativity for valid as compared to invalid trials. This effect continued to be present in the N140 time window (120-165 ms poststimulus) at ipsilateral and midline sites as well as at contralateral electrodes, all $F(1,11)>12.9$, all $p<.01$. Here, a Trial Type $\times$ Electrode Site interaction was present at midline, $F(2,22)=5.7, \quad p<.02, \varepsilon=.80, \quad$ contralateral, $\quad F(3,33)=7.1$, $p<.01, \varepsilon=.70$, and ipsilateral electrodes, $F(3,33)=4.9, p<.03$, $\varepsilon=.53$, but follow-up analyses confirmed the presence of significant attentional enhancements of N140 amplitudes at all electrode sites (main effects of trial type: all $F[1,11]>12.0$, all $p<.02$ ).

Main effects of trial type were still present between 175 and $285 \mathrm{~ms}$ after stimulus onset at ipsilateral, midline, and contralateral electrodes, all $F(1,11)>12.9, p<.01$, reflecting the later phase of the enhanced negativity on valid trials shown in



Figure 1. Left panel: Mean RTs and standard errors to tactile target stimuli on valid, invalid, and neutral trials. Right panel: Mean costs (invalid minus neutral trials), benefit (neutral minus valid trials), and total attention directing effect (invalid minus valid trials) with corresponding standard errors. 


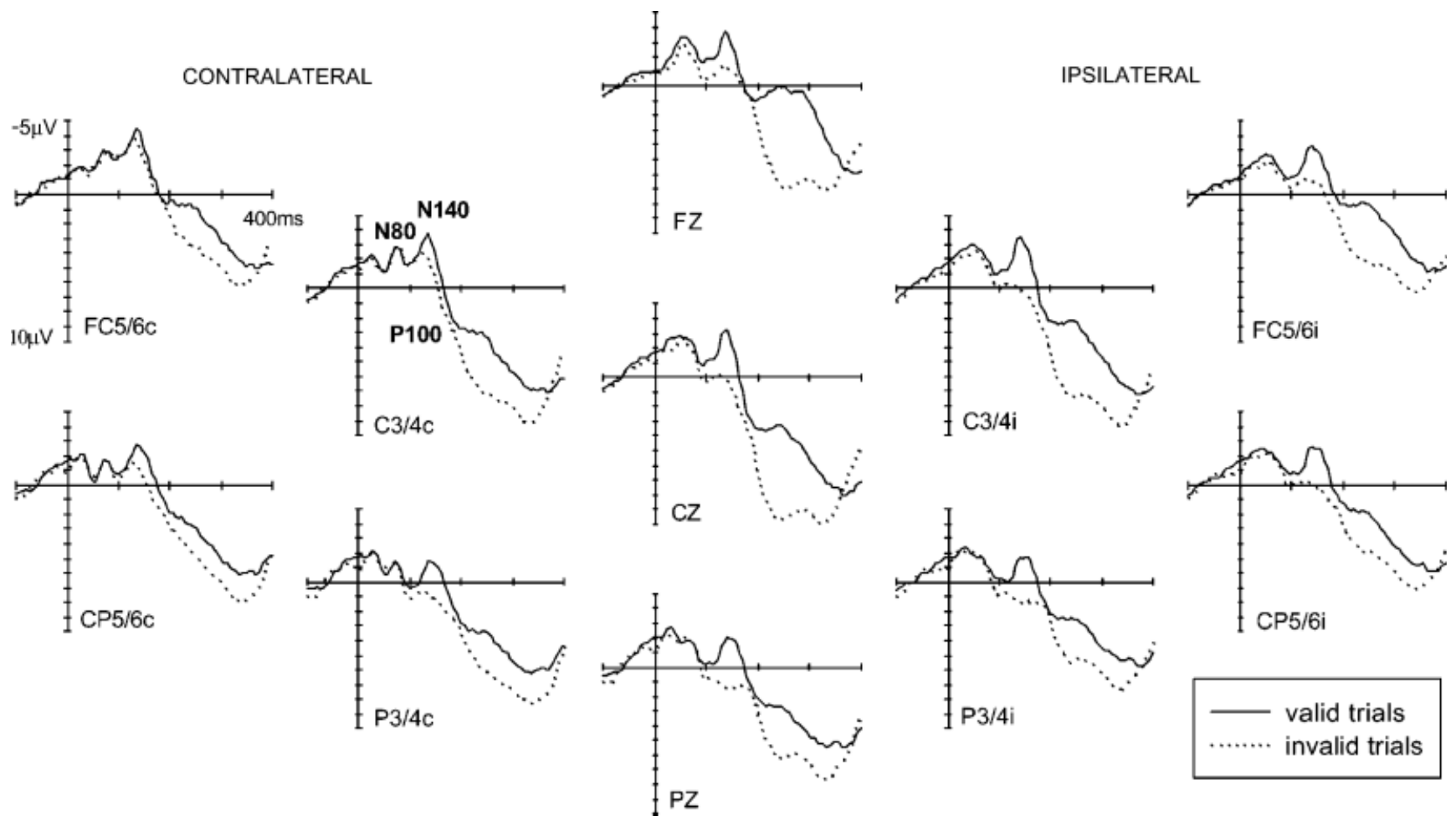

Figure 2. Grand-averaged ERPs to tactile nontarget stimuli elicited on valid (solid lines) and invalid (dotted lines) trials in the 400-ms interval following stimulus onset over the hemisphere contralateral and ipsilateral to the stimulated hand and at midline electrodes.

Figure 2. Again, Trial Type $\times$ Electrode Site interactions were observed for midline, $F(2,22)=7.7, p<.01, \varepsilon=.68$, contralateral, $F(3,33)=3.7, p<.04, \varepsilon=.71$, and ipsilateral electrodes, $F(3,33)=9.0, p<.01, \varepsilon=.60$, but follow-up analyses confirmed the presence of significant attentional negativities at all electrode sites, all $F(1,11)>7.3$, all $p<.03$.

Benefits of tactile-spatial attention: Valid and neutral trials. To assess any benefits on somatosensory processing resulting from shifts of spatial attention, SEPs on valid trials were compared to SEPs on neutral trials. Figure 3 shows SEPs in response to tactile nontarget stimuli at midline, ipsilateral, and contralateral electrode sites on valid trials (solid lines) and neutral trials (dashed lines). Benefits appear to be primarily reflected in an enhanced N140 component elicited by tactile stimuli on valid compared to neutral trials. No significant effects of trial type (valid vs. neutral) were present for the time window of the P100 component (80$120 \mathrm{~ms}$ after stimulus onset). In the N140 time range (120-165 ms after stimulus onset), a significant main effect of trial type was present at ipsilateral electrodes, $F(1,11)=13.7, p>.01$, and this effect was almost significant at midline electrodes, $F(1,11)=4.3$, $p=.06$, reflecting enhanced N140 amplitudes on valid relative to neutral trials. A Trial Type $\times$ Electrode Site interaction was present at midline electrodes, $F(2,22)=4.2, p<.04, \varepsilon=.82$, and follow-up analysis showed a significant effect of trial type on N140 amplitudes only at Pz, $F(1,11)=7.3, p<.03$.

At longer latencies (175-285 ms after stimulus onset), no main effects or interactions involving trial type were present. However, the ERP waveforms shown in Figure 3 suggest that an enhanced negativity for valid relative to neutral trials may have been present during the early part of this measurement window. This was confirmed in post hoc analyses, which were conducted on ERP mean amplitudes obtained for a narrowed latency window (180-235 ms after stimulus onset) Here, significant effects of trial type were present at ipsilateral sites, $F(1,11)=10.7, p<.01$, as well as at midline electrodes, $F(1,11)=5.41, p<.04$.

Costs of tactile-spatial attention: Neutral and invalid trials. To assess any costs on somatosensory processing resulting from shifts of spatial attention, SEPs on neutral trials were compared to SEPs on invalid trials. Figure 4 shows SEPs in response to tactile nontarget stimuli at midline, ipsilateral, and contralateral electrode sites on neutral trials (dashed lines) and on invalid trials (dotted lines). Substantial costs appear to be present for the N140 component, as well as at longer latencies. Relative to neutral trials, N140 amplitudes were attenuated on invalid trials, and longer latency ERPs were more negative on neutral as compared to invalid trials. No significant effects of trial type (neutral vs. invalid) were present for P100 latency range. In contrast, significant trial type effects were obtained for the N140 measurement window (120-165 ms after stimulus onset) at ipsilateral, contralateral, and midline electrode sites, all $F(1,11)>7.9$, all $p<.03$, reflecting a reduction of N140 amplitudes on invalid relative to neutral trials (see Figure 4).

At longer latencies (175-285 ms after stimulus onset), significant main effects of trial type were again present at ipsilateral, contralateral, and midline electrode sites, all $F(1,11)>17.3$, all $p<.01$, reflecting an enhanced negativity for stimuli on neutral compared to invalid trials. A Cue Type $\times$ Electrode Site interaction was present for ipsilateral electrodes, $F(3,33)=4.8$, $p<.03, \varepsilon=.51$, but follow-up analyses showed significant effects of trial type at all ipsilateral electrodes, all $F_{\mathrm{s}}(1,11)>11.5$, all $p<.01$. 


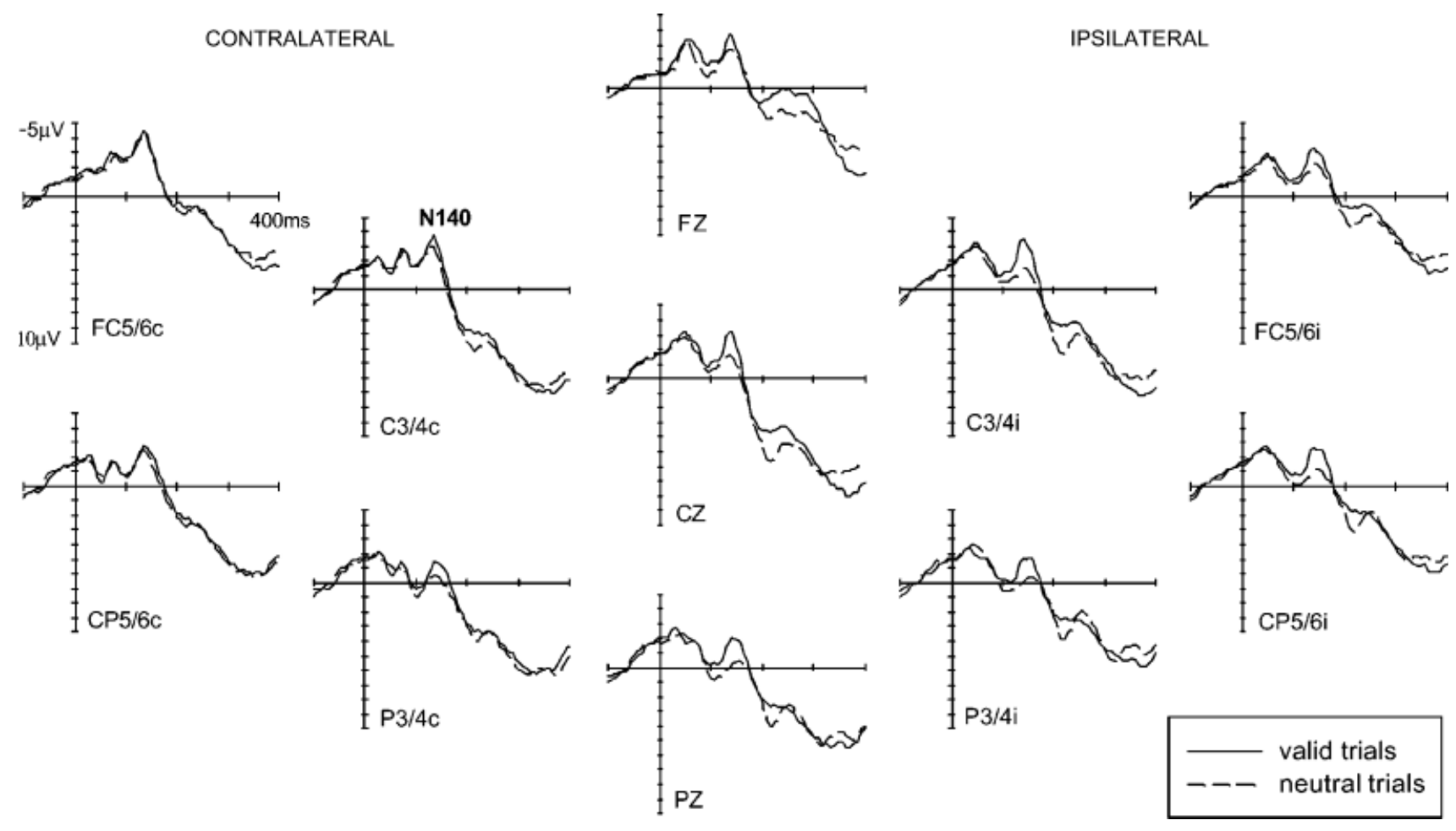

Figure 3. Grand-averaged ERPs to tactile nontarget stimuli elicited on valid (solid lines) and neutral (dashed lines) trials in the 400-ms interval following stimulus onset over the hemisphere contralateral and ipsilateral to the stimulated hand and at midline electrodes.

Cost-benefit analyses. The above analyses have shown that attentional modulations of N140 amplitudes represent costs as well as benefits of tactile-spatial attention, whereas longer latency effects appear to primarily reflect processing costs. To further assess the relative contributions of costs and benefits, these were quantified separately by subtracting ERPs on valid trials from ERPs on neutral trials (to obtain a measure of processing benefits), and by subtracting ERPs on neutral trials from ERPs on invalid trials (to measure processing costs). The resulting difference waveforms are shown in Figure 5, where the overall attention effects (valid minus invalid trials; solid lines) are plotted together with difference waves representing processing costs (neutral minus invalid trials; dotted lines) and processing benefits (valid minus neutral trials; dashed lines). Two successively elicited modulations are clearly visible in these difference waves, which correspond to attentional enhancements of N140 amplitudes and a subsequent sustained attentional negativity.

ERP difference amplitudes obtained for costs and benefits in the N140 time range (between 120 and $165 \mathrm{~ms}$ after stimulus onset) and at longer latencies (175-285 ms poststimulus) were analyzed for the factors costs/benefits (neutral minus invalid vs. valid minus neutral difference waves), stimulated hand, and electrode site. No effects involving costs/benefits were obtained in the N140 time window, thus suggesting that costs and benefits contribute equally to the attentional modulation of N140 amplitudes (see also Figure 5). In contrast, significant main effects of costs/benefits were obtained in the longer latency analysis interval at contralateral, ipsilateral, and at midline electrode, all $F(1,11)>11.2$, all $p<.01$. This observation reflects the fact that the sustained attentional negativity observed for valid relative to invalid trials at longer latencies (Figure 5, solid lines) predominantly reflects costs (Figure 5, dotted lines), but only to a small degree benefits (Figure 5, dashed lines) of spatial attention on somatosensory processing.

\section{Discussion}

The aim of the present experiment was to investigate the mechanisms underlying selective attentional processing in touch. Tactile stimuli were delivered to the left or right hand following informative and neutral cues. Informative cues provided information about likely target stimulus locations and thus were expected to induce shifts of tactile attention, whereas neutral cues were giving no information about the subsequent stimulus location. Although previous experiments have shown that directing tactile attention to one hand versus another facilitates behavioral performance (Sathian \& Burton, 1991; Spence et al., 2000) and modulates somatosensory processing (Desmedt \& Robertson, 1997; Eimer \& Forster, 2003; Forster \& Eimer, 2004; GarcíaLarrea et al., 1995; Michie, 1984; Michie et al., 1987), none of these studies have investigated whether these attentional effects primarily reflect processing benefits on valid trials where tactile stimuli are delivered to the cued (attended) hand, processing costs on invalid trials where tactile stimuli are delivered to the uncued (unattended) hand, or a mixture of both benefits and costs. To assess the question of whether the processing of tactile information is enhanced at attended locations (benefits), suppressed at unattended locations (costs), or both, we compared RTs and ERP waveforms for valid and invalid trials with neutral trials. Overall effects of attention were measured by contrasting valid versus invalid trials, whereas processing benefits and costs were assessed by contrasting valid versus neutral trials and neutral versus invalid trials, respectively. In addition to the relative 
CONTRALATERAL

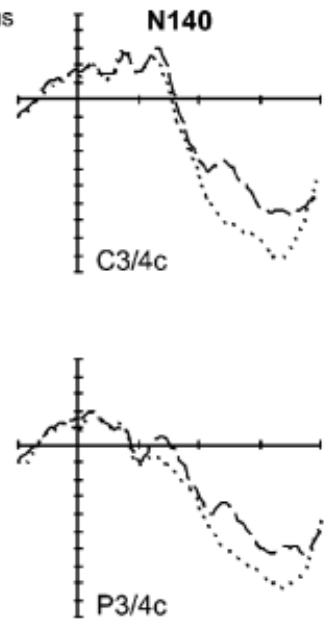
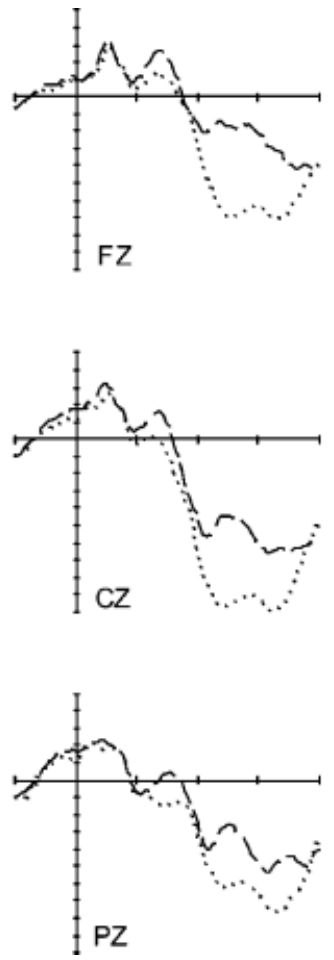

IPSILATERAL
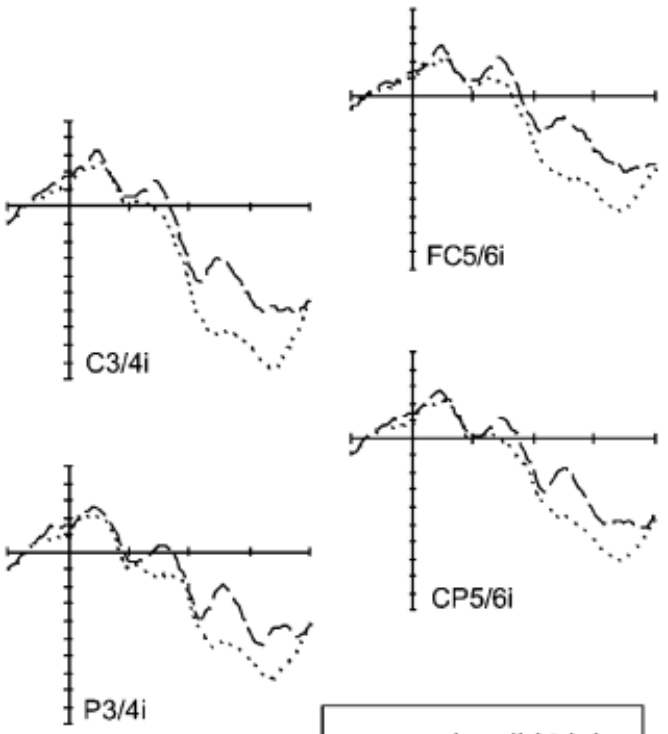

invalid trials

neutral trials

Figure 4. Grand-averaged ERPs to tactile nontarget stimuli elicited on neutral (dashed lines) and invalid (dotted lines) trials in the 400-ms interval following stimulus onset over the hemisphere contralateral and ipsilateral to the stimulated hand and at midline electrodes.

contributions of benefits and costs, the time course of costs and benefits on somatosensory processing was also investigated.

The behavioral results obtained in the present study clearly showed that both costs and benefits contribute to the overall effects of tactile-spatial attention on performance. Participants were instructed to direct their attention to the cued hand in anticipation of a tactile target stimulus, which was more likely to be presented to the hand indicated by the cue. While informative precues indicated the location of any upcoming tactile target stimulus with $80 \%$ validity, they were uninformative with respect to the location of task-irrelevant tactile nontargets. This procedure was adopted in order to obtain sufficiently large numbers of valid and invalid nontarget trials as the basis for computing somatosensory ERPs, while at the same time providing an adequate incentive for participants to shift their attention to the cued side. The fact that vocal RTs were more than $144 \mathrm{~ms}$ faster on valid relative to invalid trials demonstrated that these probability manipulations were highly effective in manipulating tactile-spatial attention. When comparing the costs (neutral vs. invalid trials) and benefits (costs minus neutral trials) of tactile attention on RTs, we found that both costs and benefits were significant, but that costs (104 ms) were larger than benefits (40 ms). This new finding that directing tactile attention to one hand versus the other results in significant performance benefits as well as in even larger performance costs (see Figure 1) is in line with earlier informal observations reported by Posner (1978), and suggests that effects of tactile-spatial attention are mediated both by a facilitation of somatosensory processing at attended locations and by a suppression of stimulus processing at unattended locations. This conclusion is further supported by the ERP results obtained in the present study.

In line with previous ERP studies on tactile spatial attention (Desmedt \& Robertson, 1997; Forster \& Eimer, 2004), we found an enhanced negativity for attended as compared to unattended stimuli, which started in the $\mathrm{P} 100$ time range, and resulted in an attentional enhancement of the N140 component as well as in a later enhanced negativity for attended as compared to unattended tactile stimuli on valid versus invalid trials. When contrasting valid and invalid with neutral trials in order to assess the relative contribution of costs and benefits to these attentional ERP modulations, we found evidence for enhanced stimulus processing at attended locations (benefits) as well as for a suppression of processing at unattended locations (costs). Processing benefits were reflected in enhanced N140 amplitudes on valid relative to neutral trials, followed by a short-lived enhanced negativity for attended stimuli. Processing costs were reflected in an attenuation of N140 amplitudes on invalid relative to neutral trials, as well as in a suppression of the subsequently elicited sustained attentional negativity. ${ }^{1}$ When comparing the relative contributions of benefits and costs on attentional modulations of somatosensory ERPs, we found that although costs and benefits contributed equally to the effects of tactile attention on N140 amplitudes, longer latency sustained attentional modulations were largely attributable to costs (Figure 5).

Results from previous ERP experiments investigating attentional costs and benefits in vision and audition have suggested that costs and benefits affect separate and successive stages of visual and auditory processing (Golob et al., 2002; Luck, 1995; Luck et al., 1994; Schröger \& Eimer; 1997). Both in vision as well as in audition, attentional costs were found to precede benefits. In contrast, the present findings suggest that processing costs and

${ }^{1}$ Although Figures 3 and 4 suggest that some processing benefits and costs may have already been present in the P100 time range (with larger negativities for attended relative to neutral trials and for neutral relative to invalid trials), these differences were not statistically reliable. 


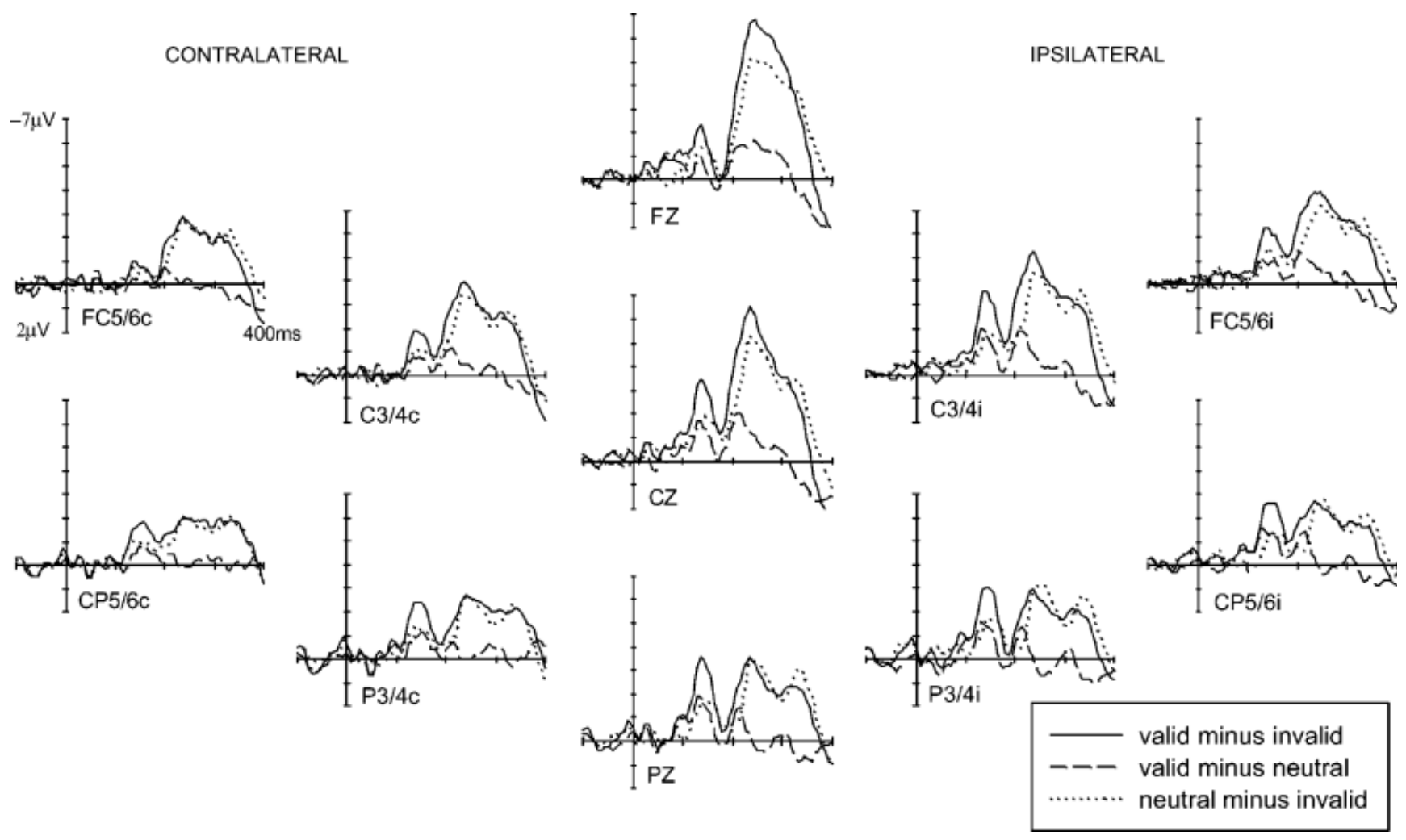

Figure 5. Difference waveforms representing the overall attention effect (valid minus invalid trials; solid lines) and the relative contributions of processing costs (neutral minus invalid trials; dotted lines) and processing benefits (valid minus neutral trials; dashed lines).

benefits are equally present at an early stage of tactile processing, and that both contribute in equal measure to the attentional modulation of the somatosensory N140 component. This apparent difference in the time course of attentional effects in touch as compared to vision and audition might indicate substantial differences in the component processes responsible for selective attentional processing between these sensory modalities. In touch, the attentional suppression of information originating from irrelevant unattended locations seems to co-occur with an attentional enhancement of sensory processing at attended locations, suggesting that both processes affect the same processing stages. However, somatosensory processes at latencies beyond the N140 component appear to be primarily affected by attentional costs (reflecting a suppression of unattended information) and not by benefits. This observation might also account for the fact that RT costs were larger than RT benefits in the present experiment.

In summary, the present study investigated the mechanisms underlying the selective attentional processing of tactile information and found new evidence based on behavioral and on electrophysiological data suggesting the presence of substantial benefits as well as costs of tactile-spatial attention on somatosensory processing. The enhancement of tactile information from currently attended locations and the suppression of information from unattended locations appears to be triggered in parallel. This suggests that in contrast to vision and audition, attentional facilitation and attentional inhibition affect the same stages of somatosensory processing.

\section{REFERENCES}

Desmedt, J. E., \& Robertson, D. (1977). Differential enhancements of early and late components of the cerebral somatosensory evoked potentials during forced-pace cognitive tasks in man. Journal of Physiology, 271, 761-782.

Eimer, M., \& Forster, B. (2003). Modulations of early somatosensory ERP components by transient and sustained spatial attention. Experimental Brain Research, 151, 24-31.

Eimer, M., Van Velzen, J., Forster, B., \& Driver, J. (2003). Shifts of attention in light and in darkness: An ERP study of supramodal attentional control and crossmodal links in spatial attention. Cognitive Brain Research, 15, 308-323.

Forster, B., \& Eimer, M. (2004). The attentional selection of spatial and non-spatial attributes in touch: ERP evidence for parallel and independent processes. Biological Psychology, 66, 1-20.
García-Larrea, L., Lukaszewicz, A. C., \& Mauguière, F. (1995). Somatosensory responses during selective spatial attention: The N120-to-N140 transition. Psychophysiology, 32, 526-537.

Golob, E. J., Pratt, H., \& Starr, A. (2002). Preparatory slow potentials and event-related potentials in an auditory cued attention task. Clinical Neurophysiology, 113, 1544-1557.

Josiassen, R. C., Shagrass, C., Roemer, R. A., Ercegovac, D. E., \& Straumanis, J. J. (1982). Somatosensory evoked potential changes with a selective attention task. Psychophysiology, 19, 146-159.

Luck, S. J. (1995). Multiple mechanisms of visual-spatial attention: Recent evidence from human electrophysiology. Behavioural Brain Research, 71, 113-123.

Luck, S. J., Hillyard, S. A., Mouloua, M., Woldorff, M. G., Clark, V. P., \& Hawkins, H. L. (1994). Effects of spatial cuing on luminance 
detectability: Psychophysical and electrophysiological evidence for early selection. Journal of Experimental Psychology: Human Perception and Performance, 20, 887-904.

Michie, P. T. (1984). Selective attention effects on somatosensory eventrelated potentials. Annals of the New York Academy of Science, 425, $250-255$.

Michie, P. T., Bearpark, H. M., Crawford, J. M., \& Glue, L. C. T. (1987). The effects of spatial selective attention on the somatosensory event-related potential. Psychophysiology, 24, 449-463.

Posner, M. I. (1978). Chronometric explorations of mind. New York: Laurence Erlbaum Associates, Inc.

Sathian, K., \& Burton, H. (1991). The role of spatially selective attention in the tactile perception of texture. Perception \& Psychophysics, 50, 237-248.
Schröger, E., \& Eimer, M. (1997). Endogenous covert spatial orienting in audition: "Cost-benefit" analyses of reaction times and event-related potentials. The Quarterly Journal of Experimental Psychology, 50A, 457-473.

Spence, C. J., \& Driver, J. (1997). Audiovisual links in endogenous covert spatial attention. Journal of Experimental Psychology: Human Perception and Performance, 22, 1005-1030.

Spence, C. J., Pavani, F., \& Driver, J. (2000). Crossmodal links between vision and touch in covert endogenous spatial attention. Journal of Experimental Psychology: Human Perception and Performance, 26, 1298-1319.

(Received August 3, 2004; AcCePted October 19, 2004) 